\title{
Structural synthesis as method of zone-phase controller modernization
}

\author{
Vladlen Ivanov \\ Novosibirsk State Technical University \\ Novosibirsk, Russia \\ NSTU \\ ivanov.etk@yandex.ru \\ Andrei Kapustin \\ Novosibirsk State Technical University \\ Novosibirsk, Russia \\ NSTU \\ kapusta_nsk@mail.ru
}

\author{
Sergei Myatez \\ Novosibirsk State Technical University \\ Novosibirsk, Russia \\ NSTU \\ serg_y_7578@mail.ru \\ Evelina Langeman \\ Novosibirsk State Technical University \\ Novosibirsk, Russia \\ NSTU \\ langeman.evelina@gmail.com
}

\author{
Irina Alekseeva \\ Novosibirsk State Technical University \\ Novosibirsk, Russia \\ NSTU \\ alekseeva1201@mail.ru
}

\begin{abstract}
The tasks of structural synthesis and circuitry analysis of static converters are always fraught with difficulties in solving, because they contain elements with non-linear voltampere characteristics and allow a huge number of alternatives fixed relationships combinations (topological structures) between the individual elements. There are many circuit designs for semiconductor rectifiers with acceptable power characteristics for controlled three-phase alternating current rectifiers, but there are not many variants for controlled single-phase AC rectifiers. The circuit design of almost all known rectifier was obtained by heuristic way.
\end{abstract}

Keywords - Method, structural synthesis, rectifier.

\section{INTRODUCTION}

Systems engineering methods and circuit-based synthesis of zone phase controllers are quite complex due to the valve specificity as a nonlinear element. In addition there is a variety of semiconductor converters' equivalent circuit for different modes and operation intervals. Despite the objective difficulties of new formal synthesis methods, several converter classes have received the circuit development using matrixtopological structural synthesis methods (based on the graph of state changes; based on the main topological matrix). The converter circuit designs of certain classes are obtained by algebraic (based on tensor methodology) and aggregatemodular approaches to the synthesis [6].

However, methods of the simplest conversion circuit's creation which are presented above are too difficult despite the opportunity to make the convertor schematic solutions by means of them. These methods contain a large number of mathematical calculations due to the large number of element types, including reactive ones, and the complexity of the transformative processes in the most synthesized converters. But a large number of mathematical calculations decrease the number of heuristic type operations.

There are cases of incorrect circuit synthesis. These methods are actively used for many converter classes due to the large number of initial, intermediate and result parameters. For the rectifier circuits synthesis the source parameters are supply network parameters, and that is enough for these methods. However, for the specified result parameters, such as pulse number, the rectified voltage level and load current value it is almost impossible to identify intermediate parameters. These parameters take into account the number of serial or parallel conversion cascades, the configuration of secondary winding circuits and rectification circuit, installed power, which could contribute to the above methods application [6].

The uncertainty of the input data of the rectifiers contributes an inaccurate circuit description in terms of classical algebraic approach.

At this point the creation of rectifier's schemes with defined properties is based on the method of a new rectifier's schemes aggregation, by merging of already known schemes. Evidence-based theoretical result of the structural synthesis methods of rectifiers' converters schemes with natural commutation is not selected in a separate research area. There is no deeply developed basic methodology of circuit design formalization methods for natural commutated rectifiers. Current studies are focused on the modernization and energy 
performance improvement of bridge circuits with 6 and 12 pulse rectifiers, which have suspended the development of efficient rectifiers with the number of pulses over twelve. Some exceptions are the inventions of high voltage rectifier algorithms with multistage build of rectifier's branch [7].

However, the ability of rectifier circuit design based on two element types ( an idealized valve and valve winding, which is provided by ideal source) without taking into account the impact of the accumulation and loss of energy processes, leads to the idea of finding out more simple methods of structural synthesis.

\section{GENERAL PROVISIONS ON STRUCTURAL SYNTHESIS}

Circuit synthesis consists of system analysis, which defines the rectifier structure and circuit analysis that approximately calculates of synthesized circuits. Modern single phase zone converters are based on equivalent single-phase rectification circuits based on known circuits. So, single-phase bridge controlled rectifier is a basis for more complex phase converters design. Mainly at the inventive stage, new circuit designs are intuitive. Any voltage system could be represented as discrete one but also moving on vector system potential plane.

It should be considered that system depends on the rectification frequency and should be consistent with the equality of rectified voltage result radius vectors. In vector systems fixed positions irrespectively of the voltage results formation mode and the systems number of secondary windings voltage involved in the transformation process, all one-way valve connections between the working points of feeding systems and DC load are identified as well as communication systems among themselves, if the transformation process involves more than one system. Obtained connections define converter circuit topology. It was found that for synthesis based on naturally electric connections determination, vectors and time diagrams of secondary winding voltages could be used [6].

The main task of the electrical energy converter is a communication between two or more electrical systems and changing the parameters of electrical energy to ensure the exchange between systems. According to this definition, the rectifier task is to connect $\mathrm{AC}$ and DC systems, where the parameters of electrical energy of associated systems, which allows the exchange or transfer of electric energy to be transformed. Thus, the function of the converter, in fact, is the matching and changing sequence in relationships between the individual conductors of associated systems determines the function of the converter as a rectifier [6].

At the stage of circuit-based synthesis, this does not take into account any compensatory parameters adjustment of electromagnetic processes, any converter need to be idealized. It means to neglect the possibility of electrical energy accumulation in it or converting it into other kinds of energy. In this case, the structure of the converter will consist of switching idealized elements.

\section{THE CONSTRUCTION OF A SINGLE-PHASE ZONE RECTIFIER WITH AN INCREASED POWER FACTOR}

Making circuit design solution of single-phase alternating rectifier with high power performance indicators is getting complicated by single-phase alternating current system which is always unbalanced in comparison with a three-phase system. Due to this taking into consideration generally accepted assumptions of static converter ideality elements [3], the formal analysis shows that the three-phase alternating rectifier for inductive load enables one to provide the input power factor:

$$
\chi=\frac{3}{\pi} \approx 0,95
$$

while the single-phase alternating current rectifier provides:

$$
\chi=\frac{2 \sqrt{2}}{\pi} \approx 0,9
$$

In this context, the value $\chi$ of controlled rectifier appears to be much dependant on the value of artificial delay of rectifier power semiconductor devices opening (PSD) (commutation delay angle) introduced for the possibility of regulation of the rectified voltage value $\left(U_{d}\right)$. Thus, for the controlled single-phase alternating current rectifier with zero diode operating for the active-inductive load, the value $\chi$ is defined by [4]:

$$
\chi=\frac{(1+\cos (\alpha))}{\sqrt{(1+\cos (\alpha))^{2}+\sin ^{2}(\alpha)}} \cdot \frac{2 \sqrt{1+\cos (\alpha)}}{\sqrt{\pi} \sqrt{\pi-\alpha}},
$$

where $\alpha$ is an artificial delay of PSD opening.

The synthesis method peculiarity of single-phase alternating current rectifier will be each resulted rectified voltage that should be considered as maximum electric potential difference on topographic potential plane containing time base sweep of voltage is represented in the form of a vector diagram. In this case all modification accessible voltage vectors will appear to be collinear due to the single system peculiarity background.

It is also accepted, for synthesis convenience, that the magnitude of voltage vector corresponds to the secondary transformer winding that produce them and reflects their peak value.

It is appropriate to consider the examples of the method illustration and more simple application of its specifications.

Let it be required to put into action unidirectional conductance (rectifying) of single-phase alternating current. For this reason let us imagine that secondary winding vector is against its own inactive output. The second active output of the winding is connected to PSD represented by VD diode (Figure 1) to ensure unidirectional conductivity. 


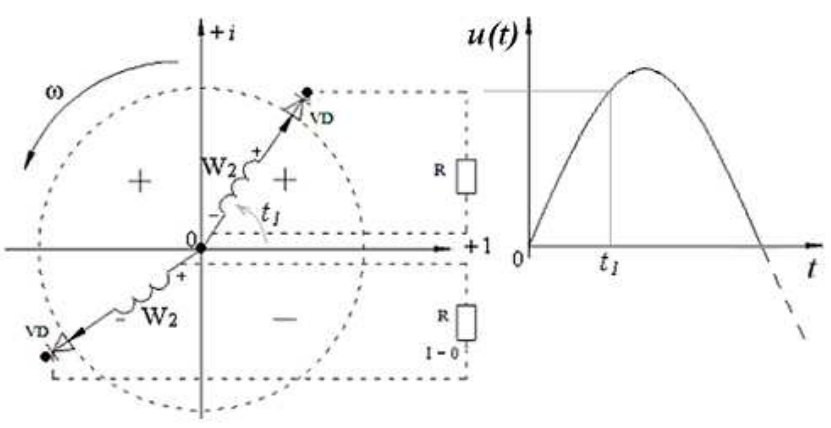

Fig. 1. Relationship (connection) of topology rectifier elements with time base sweep vector diagram on topographic potential plane.

As the result, it is getting obvious that only within the time interval when no connection winding end induces positive Electromotive Force (EMF), VD diode passes current but the secondary winding $\mathrm{W}_{2}$ works.

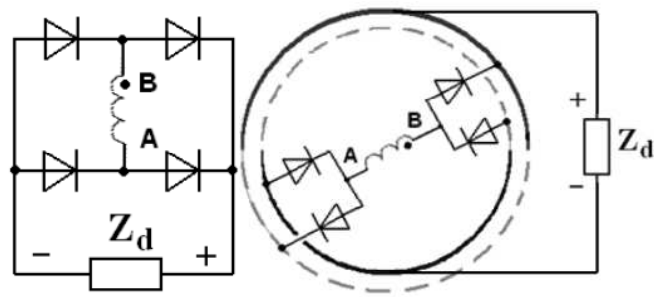

Fig. 2. Presentation of bridge circuit of rectified single-phase alternating current

One of important stages of the rectifier's circuit synthesis is to find the most rational valve structure and phase number convertor (PNC) windings connections, when a rectified voltage curve will have the required pulsations number per the supply voltage period. In general, the required pulsations number could be obtained by an infinite ways number of the windings and valves connection. However, during the targeted synthesis the main requirements for rectifier circuit are requirements, which provide: a minimum installed capacity of transformer equipment; minimal power losses in valves; performing functional task by device with required quality conversion.

Thus, we can get more advanced rectified circuit (Figure 2), known as Graetz-Pollack single-phase bridge circuit [2].

Having replaced diodes by controlled PSD and having set up, for instance, thyristors, we can get controlled bridge rectifier of single-phase alternating current with phased regulation of rectified voltage (Figure 6).

Applying a signal opening towards PSD with the delayedaction at angle $\alpha$, we are getting a picture of accessible potentials of a projection of diagonal maximum difference of which is time-base swept and will give a typical oscillogram of voltage output (or current, in case of active load) halfperiod average value of which is regulated by value change of $\alpha[3]$.

To retain the possibility of controlling the value of rectified voltage, simultaneously improving its shape (it will reduce percentage composition of upper harmonics and improve $\chi$ indicator) the zone-phase rectifiers are used in practical work. They combine the technique of phase control by means of amplitude connection by means of PSD additional secondary winding.

Consequently, it is sufficient to combine several bridge rectifiers to build a simple zone-phase regulator. Using aggregation as the most common approach in the structural synthesis method given at Figure 3, a two stage single-phase rectifier was produced. This is the result of combination (aggregation) of already known circuits. Figure 3 in assumption of active load availability shows the curves: 1 first step (zone/stage) and 2 - second step (zone/stage) of rectified voltage (or current).

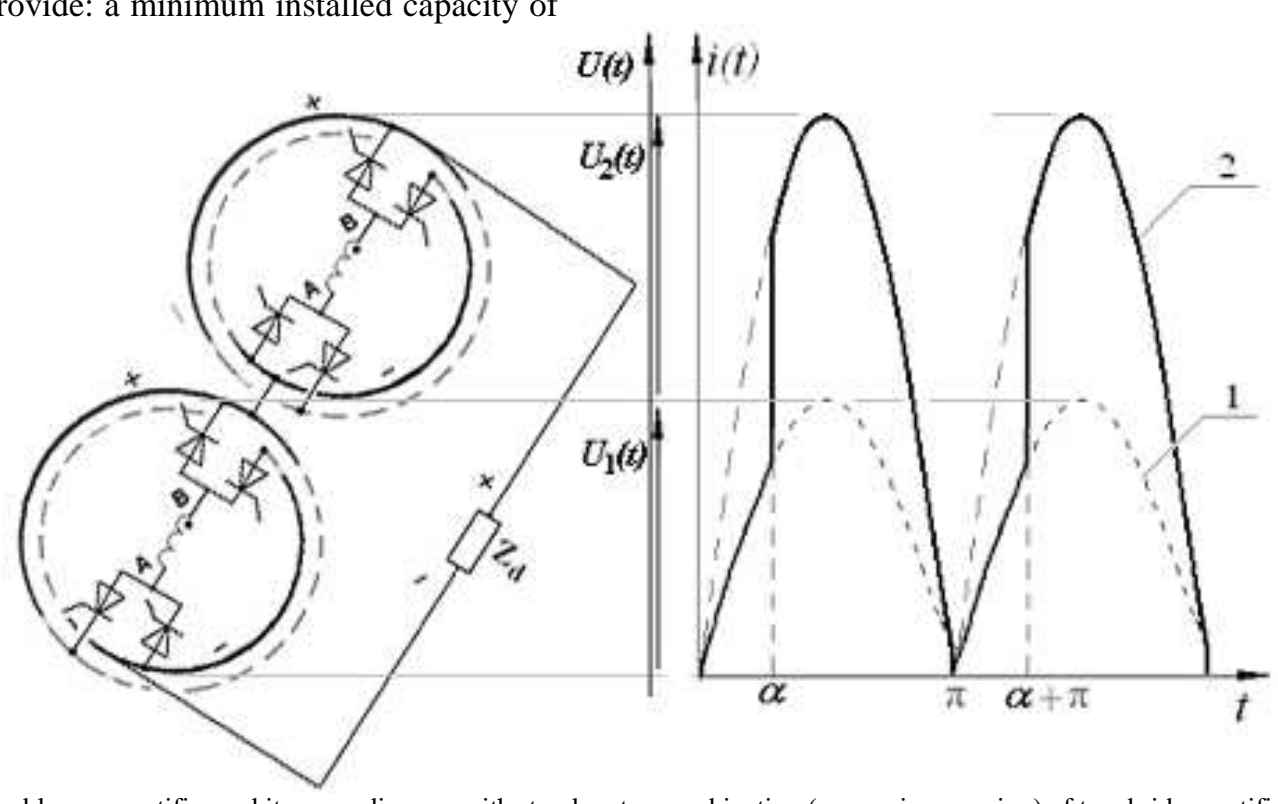

Fig. 3. Production of double zone rectifier and its wave diagram with step-by-step combination (successive merging) of two bridge rectifiers of single-phase alternating current. 
In Figure 3, the schematic circuit diagram can be produced in dual zone execution and, in practice it is known as the Ogier circuit [7].

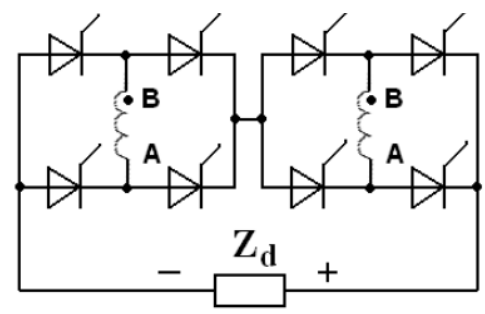

Fig. 4. Ogier schematic circuit diagram in dual zone execution.

A simple analysis demonstrates that such circuit has double number of PSD which are step-by-step connected on the way of the rectified current flow. It doubles the capacity losses, decreases $\eta$ value and restricts practical application.

To decrease capacity losses a number of PSD needs to be reduced being connected on the way of rectified current flow resulted in preserving of the potential distribution of dual zone rectifier on topographic plane. Such zones combination is feasible, for example, through their combination as it is shown in Figure 5.

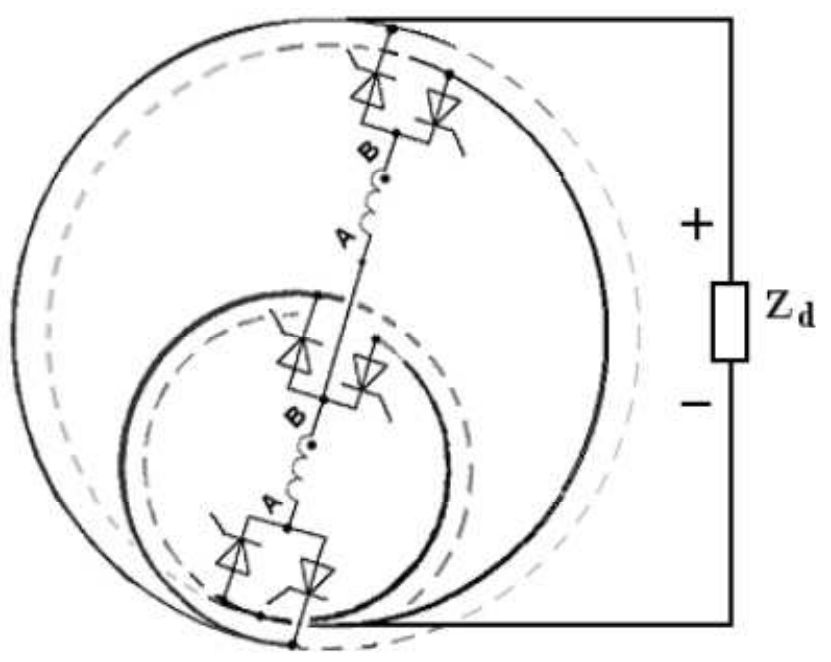

Fig. 5. Production of dual zone rectifier through combination (merging) of two zones by locus potential distribution of rotating vectors - windings.

Due to combination (merging), we can significantly reduce half of PSD number, which are step-by-step connected on the way of rectified current flow. This will be ensured by increasing of $\eta$ value by value of reduced capacity losses in PSD (in practice $\eta$ is increased at an average of $3-5 \%$ based on real variables of feeding transformer). The rectifier in Figure 5 (its schematic circuit is shown in Figure 6) was widely practiced and known as bridge zone-phase rectifier. [5].

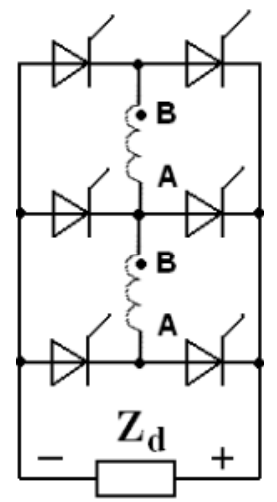

Fig. 6. Schematic circuit diagram of bridge zone-phase rectifier in dual zone execution.

\section{CONCLUSIONS}

Thus, simple regulations of the structural synthesis method can be placed onto controlled and uncontrolled rectifiers of a single-phase alternating current.

Obtained connections define the converter circuit topology. It was found that for synthesis based on naturally electric connections determination, vectors and time diagrams of secondary winding voltages can be used.

Simultaneous accomplishment of all the requirements by a converting device is virtually impossible. This complicates the synthesis formalization and predetermines heuristic search extension, simultaneously encouraging researchers to search system synthesis methods, allowing formalizing or simplifying the synthesis.

The method of structural synthesis will have the greatest effect in combination with well-known analytical methods providing quantitative assessment of the highest peak of perfection of obtained circuit design solutions.

\section{REFERENCES}

[1] S.A. Yevdokimov, G.S. Zinovyev, "Rectifiers classification option per topological features", Current issues of electronic instrument making: IXth International Conference Proceedings, Novosibirsk: NSTU Publisher, V.7, pp. 3-14. 2008.

[2] S.A. Yevdokimov, N.I. Shchurov, Structural synthesis of multi-phase valve inverters, Novosibirsk: NSTU Publisher, 2010, p. 423.

[3] G.S. Zinovyev, Power electronics, Moscow, Yurait, 2016

[4] M.M. Djaborov, S.V. Myatezh, N.I. Shchurov, "Itinerant Phase Converter with Improved Energy Performance", Applied Mechanics and Materials, Vol. 792, pp. 183-188, 2015.

[5] A.V. Plaks, Control systems of electric rolling stock, M.: Training Center for Education in rail transport, 2005, p. 360.

[6] S.A. Yevdokimov, N.I. Shchurov, S.V. Myatezh, O.L. Volkova, A.A Stepanov, "Research of four zone rectifier",Transport: science, engineering, management: Scientific Information Collection, M.: VINITI, №12, pp. 25-29, 2011.

[7] M.A. Sleptsova, Fundamentals of electric transport: textbook for students of higher educational institutions under the General editorship, M.: Academy, p. 464, 2006

[8] M.M. Dzhaborov, S.V. Myatezh, V.V. Ivanov, "Research of four-zone converter in regenerative braking mode", Applied Mechanics and Materials, Vol. 698, Electrical Engineering, Energy, Mechanical Engineering, EEM - 2014, pp. 101-105. 2015. 
[9] M.M. Djaborov, S.V. Myatezh, N.I. Shchurov, "Single - phase inverter of alternating current to direct one", patent. Russian Federation RU №148770, MPK: H02M 7/155, Bulletin № 35, 2014.

[10] V.P. Klimov, "Modern directions of the development of AC power converters", Practical power electronics, No.25, pp. 43-51, 2007.

[11] S. Reznikov, V. Bocharov, Ye. Parfenov, N. Gurenkov, A. Kornilov, "Electric and electromagnetic compatibility of secondary impulse power sources with autonomous AC power supply systems" , Power electronics, No.5, pp. 86-89, 2009.

[12] V.A. Aleshkevich, University course of general physics. Electromagnetism, Moscow: Fizmatlit, 2014.

[13] V. V. Ivanov, S. V. Myatez, A. V. Kapustin, I. K. Alekseeva, "Development prospects of single-phase zone rectifiers", The 11 International forum on strategic technology (IFOST 2016), Novosibirsk : NSTU, Pt. 2, pp. 105-107, 2016.

[14] G.S. Zinovev, Bases of the power electronics, Novosibirsk, NSTU, 2003, p. 664.

[15] V. V. Ivanov, S. V. Myatez, E. G. Langeman, N. I. Schurov, "Pulse-width control in ladder structure four-phase rectifier for AC-electromotive," IOP Conf. Series: Materials Science and Engineering, 127(1) 012004. 2016.

[16] B.G. Yuzhakov, Electric drive and converters of rolling stock: Textbook for technical schools and colleges of rail transport, M.: SEI Educational Centre for Education in rail transport, 2007, p. 396.

[17] L.A. Bessonov, Theoretical Foundations of Electrical Engineering, Electrical circuits: Ucheb.dlya university students, M .: Gardariki, 1987, p. 293. 